Cite this: RSC Adv., 2017, 7, 52238

Received 23rd September 2017 Accepted 3rd November 2017

DOI: 10.1039/c7ra10556h

rsc.li/rsc-advances
Check for updates

\section{Controlled synthesis of $\mathrm{EuPO}_{4}$ nano/ microstructures and core-shell $\mathrm{SiO}_{2} \mathrm{CEuPO}_{4}$ nanostructures with improved photoluminescence $\uparrow$}

\author{
Kui-Suo Yang, ${ }^{a}$ An-Ping Wu, ${ }^{a}$ Xin Zhao, ${ }^{a}$ Yu Yan, ${ }^{a}$ Xue-Yuan Guo, ${ }^{a}$ Yu-Long Bian, ${ }^{a}$ \\ Jin-Rong Bao, (D) *a Wen-Xian $\mathrm{Li}^{\mathrm{a}}$ and Xiao-Wei Zhu*b
}

\begin{abstract}
Cluster-like, flower-like and sphere-like EuPO ${ }_{4}$ nano/microstructures and uniform core-shell $\mathrm{SiO}_{2} \mathrm{QEuPO}_{4}$ nanostructures have been controllably synthesized by the hydrothermal route and co-precipitation method, respectively. The as-synthesized products are characterized by means of X-ray diffraction (XRD), scanning electron microscopy (SEM), transmission electron microscopy (TEM), energy-dispersive spectroscopy (EDS), X-ray photoelectron spectroscopy (XPS) and infrared spectroscopy (IR). The possible formation mechanism of the as-synthesized products is proposed. The photoluminescence properties demonstrate that the locations of the strongest peaks of nano/microstructured $\mathrm{EuPO}_{4}$ and core-shell nanostructured $\mathrm{SiO}_{2}\left(\mathrm{aEuPO}_{4}\right.$ are different. In the emission spectrum of the core-shell $\mathrm{SiO}_{2}\left(\mathrm{EuPO}_{4}\right.$ nanostructures, the ${ }^{5} D_{0} \rightarrow{ }^{7} F_{2}$ transition is much stronger than the ${ }^{5} D_{0} \rightarrow{ }^{7} F_{1}$ transition, indicating that $\mathrm{Eu}^{3+}$ ions occupy low symmetry sites in the $\mathrm{EuPO}_{4}$ lattice. Furthermore, the core-shell $\mathrm{SiO}_{2}\left(\mathrm{aEuPO}_{4}\right.$ nanostructures have stronger emission intensity than the flower-like and sphere-like $\mathrm{EuPO}_{4}$ nano/ microstructures.
\end{abstract}

\section{Introduction}

Core-shell nanomaterials have attracted significant attention due to their various unique properties, including optical, ${ }^{1}$ electrical, ${ }^{2}$ magnetic, ${ }^{3}$ thermal, ${ }^{4}$ and mechanical $^{5}$ properties. The applications of these core-shell particles are very broad because they often exhibit improved physical and chemical properties over their single-component counterparts, and include biomedical applications, catalysts and electronics. ${ }^{6-9}$ Most core and shell materials are composed of polymers, inorganic solids and metals. ${ }^{10-12} \mathrm{SiO}_{2}$ has been widely used to fabricate core-shell materials as a core due to its good properties. ${ }^{13,14}$ When $\mathrm{SiO}_{2}$ was used as the core material, the $\mathrm{Si}-\mathrm{OH}$ groups and external hydrogen bonds on the surface of $\mathrm{SiO}_{2}$ had a very strong affinity, which makes the $\mathrm{SiO}_{2}$ bond easily with a variety of materials through chemical bonds and electrostatic adsorption interactions. $\mathrm{SiO}_{2}$ core-shell nanomaterials can be used as important functional materials through various modifications with rare-earth compounds. For example, as reported in our previous work on $\mathrm{SiO}_{2} @ D y(\mathrm{MABA}-\mathrm{Si}) \mathrm{L}(\mathrm{L}=$ Phen or Dipy)

${ }^{a}$ School of Chemistry and Chemical Engineering, Inner Mongolia University, Hohhot 010021, China. E-mail: jinrongbao@imu.edu.cn; Tel: +86-0471-4992981

${ }^{b}$ College of Pharmacology, Inner Mongolia Medical University, Hohhot 010059, China. E-mail: zxwtxwd@sina.com

$\dagger$ Electronic supplementary information (ESI) available. See DOI: 10.1039/c7ra10556h core-shell nanometer luminescent composites, the emission intensity of the nanomaterials can be greatly enhanced by the formation of the core-shell structure. ${ }^{15}$ The silica-coated coreshell $\mathrm{Y}_{2} \mathrm{O}_{3}: \mathrm{Eu}^{3+}, \mathrm{Co}^{2+}$ composite particles as fluorescent contrast agents in cell imaging have been prepared by a precipitation method, and the particles with a silica core fluoresced more strongly. ${ }^{16}$ Besides, the emission intensity of core-shell $\mathrm{SiO}_{2} @ \mathrm{Y}_{2} \mathrm{O}_{3}: \mathrm{Eu}^{3+}$ particles can be tuned by the $\mathrm{SiO}_{2}$ core size and the number of coating cycles. ${ }^{17}$ It can be also mentioned that $\mathrm{SiO}_{2} @ \mathrm{LaVO}_{4}: \mathrm{Eu}^{3+}$ with tuned emission in the visible region and promising applications as color television and the high-pressure mercury lamp were obtained via sol-gel process. ${ }^{18}$ The $\mathrm{SiO}_{2}$ core-shell nanomaterials can not only significantly save the cost of expensive rare earth materials by covering them on cheap cores, but also improve the luminescence intensity of phosphor.

Recently, rare earth phosphate is considered as an important family of phosphors, due to its easy synthesis, low cost, high thermal and chemical stability over a wide range of temperature. ${ }^{19,20}$ In addition, rare earth phosphate nanomaterials have potential applications in the LCD displays, plasma display panels (PDPS), field emission displays, new generation fluorescent lamps, and so on. ${ }^{21-24}$ Many preparation methods have been used to fabricate nanostructured rare earth phosphates such as nanowires, nanotubes, nanoplates, and nanorods to improve the luminescence emission intensity. ${ }^{25-28}$ So far, much 
research effort has been devoted to synthesize the core-shell $\mathrm{SiO}_{2} @ \mathrm{REPO}_{4}$ phosphor materials. However, the approaches to fabricate core-shell structured rare earth orthophosphates are not mature. Yu et al. have synthesized $\mathrm{SiO}_{2} @ \mathrm{LaPO}_{4}: \mathrm{Ce}^{3+} / \mathrm{Tb}^{3+}$ particles by sol-gel method, and the emission intensity of $\mathrm{Tb}^{3+}$ increases with the increasing of $\mathrm{SiO}_{2}$ core particles size and the annealing temperature. ${ }^{29}$ The emission intensity of $\mathrm{SiO}_{2} @$ $\mathrm{LaPO}_{4}: \mathrm{Eu}^{3+}$ core-shell phosphors also increases with increasing the coating cycles. ${ }^{30}$ Therefore, it is desirable to explore feasible and easily controllable methods for the synthesis of core-shell $\mathrm{SiO}_{2} @ \mathrm{REPO}_{4}$ nanomaterials.

In this paper, we report the synthesis of cluster-like, flowerlike and spherical-like $\mathrm{EuPO}_{4}$ nano/microstructures materials by a simple hydrothermal process, in combination with the coprecipitation method to controllably synthesize the core-shell $\mathrm{SiO}_{2} @ \mathrm{EuPO}_{4}$ nanostructures. The possible formation mechanisms of the synthesized materials were proposed. Moreover, the photoluminescence properties of the as-synthesized products were compared. It is demonstrated that the core-shell $\mathrm{SiO}_{2} @ \mathrm{EuPO}_{4}$ nanostructures showed improved photoluminescence properties. The core-shell $\mathrm{SiO}_{2} @ \mathrm{EuPO}_{4}$ nanostructures phosphors can lower the cost of precious phosphors, which are promising for applications in the field of emission displays, lamps for lighting, and plasma display panels.

\section{Experimental}

\subsection{Material and reagents}

$\mathrm{Eu}_{2} \mathrm{O}_{3}$ (99.99\%), triethyl phosphate (TEP), ammonia and orthophosphate acid were all provided by Sinopharm Chemical Reagent Limited Corporation. Tetraethoxysilane (TEOS) was provided by Beijing Industry of Fine Chemical Limited Corporation. All reagents were analytical grade and used as received without further purification. The europium nitrate was prepared by dissolving $\mathrm{Eu}_{2} \mathrm{O}_{3}$ was dissolved in $10 \%$ nitric acid, followed by evaporation and drying in vacuum.

\subsection{Synthesis of $\mathrm{EuPO}_{4}$ nano/microstructures}

For the synthesis of cluster-like and flower-like $\mathrm{EuPO}_{4}$ nano/ microstructures, a certain amount of europium nitrate was dissolved in $10 \mathrm{~mL}$ of deionized water, and added into $6.0 \mathrm{~mol} \mathrm{~L}^{-1} \mathrm{H}_{3} \mathrm{PO}_{4}$ solution under stirring. Meanwhile, the reactant $\mathrm{PO}_{4} / \mathrm{Eu}$ molar ratio was changed from 60 to 200 by decreasing the amount of europium nitrate. Then the mixture solution was transferred into a $50 \mathrm{~mL}$-capacity Teflon-lined stainless steel autoclave and heated at $100{ }^{\circ} \mathrm{C}$ for $12 \mathrm{~h}$. After cooling down the autoclave to room temperature, the precipitation was separated by centrifugation, washed with deionized water and ethanol several times, and finally dried in air at $60{ }^{\circ} \mathrm{C}$ for $8 \mathrm{~h}$.

The synthesis of spherical-like $\mathrm{EuPO}_{4}$ nanostructures was achieved by setting the $\mathrm{PO}_{4} / \mathrm{Eu}$ molar ratio to be 200 . The $\mathrm{pH}$ value of the system was adjusted from 2.0 to 4.0 with ammonia (25\%) under stirring, and then transferred into a $50 \mathrm{~mL}$-capacity Teflon-lined stainless steel autoclave at $100{ }^{\circ} \mathrm{C}$ for $12 \mathrm{~h}$.

\subsection{Synthesis of the core-shell $\mathrm{SiO}_{2} @ \mathrm{EuPO}_{4}$ nanostructures}

The highly monodispersed $\mathrm{SiO}_{2}$ submicro-spheres were fabricated following the well-known Stöber method. ${ }^{31}$ First, deionized water and absolute ethanol were mixed, followed by the addition of aqueous ammonia and tetraethoxysilane. A white silica colloidal suspension was formed and stirred with $3.5 \mathrm{~h}$. The $\mathrm{SiO}_{2}$ submicro-spheres were centrifugally separated from the suspension and washed with ethanol and deionized water several times, prior to drying in air at $60^{\circ} \mathrm{C}$ for $12 \mathrm{~h}$. After that, a certain amount of $\mathrm{SiO}_{2}$ submicro-spheres is mixed with the prepared $0.1 \mathrm{~mol} \mathrm{~L}^{-1}$ europium nitrate solution. Then, $0.5 \mathrm{~mL}$ triethyl phosphate (TEP) was added under magnetic stirring at $70{ }^{\circ} \mathrm{C}$ for 3 days. The resulting solution was centrifuged and washed with ethanol and deionized water several times, respectively.

\subsection{Characterization}

The size and morphology of the products were characterized by scanning electronic microscopy (SEM; Hitachi S-4800, Japan and LEO1530, Germany) and transmission electron microscopy (TEM; FEI Tecnai F20, USA). XRD patterns were measured by a 21 kW extra power X-ray diffractometer (Model M21XVHF22, MAC science Co. Ltd., Japan) using $\mathrm{Cu} \mathrm{K} \alpha$ radiation $(k=0.1541 \mathrm{~nm})$ over a $2 \theta$ range of $10-60^{\circ}$ at room temperature. Infrared spectra (IR) of powders were examined in the range of $400-4000 \mathrm{~cm}^{-1}$ by the $\mathrm{KBr}$ pressed disc method on a Nicolet NEXUS 670 FT-IR. The photoluminescence spectra were determined on a FLS980 fluorescence photometer with the slit width of $2.0 \mathrm{~nm}$ at room temperature.

\section{Result and discussion}

\subsection{Structure and morphology of $\mathrm{EuPO}_{4}$ nano/ microstructures}

The crystal structure and the phase purity of the products were identified by the XRD. Fig. 1 shows the typical XRD patterns of

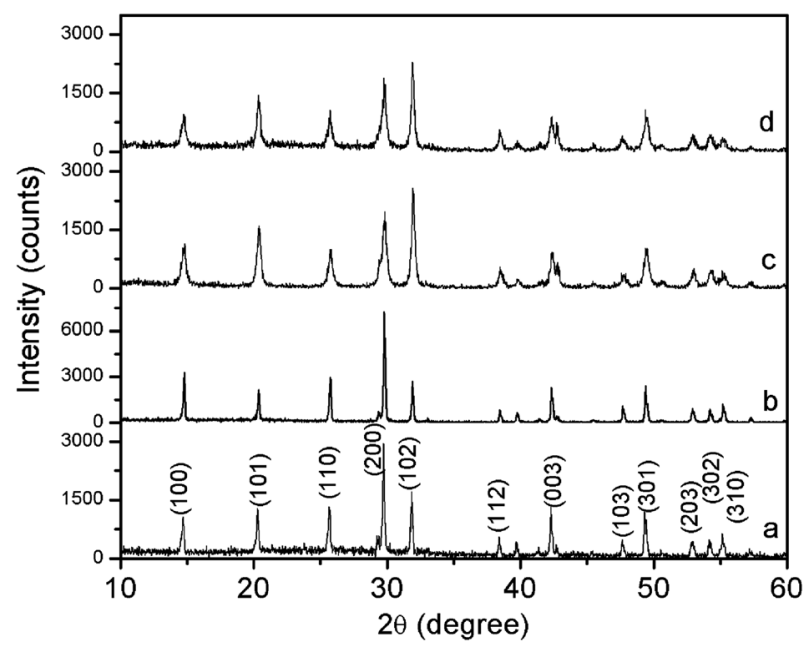

Fig. 1 XRD pattern of $\mathrm{EuPO}_{4}$ nano/microstructures prepared with the different reactant $\mathrm{PO}_{4} /$ Eu molar ratios of (a) 60, (b) 200, and different $\mathrm{pH}$ values (c) $\mathrm{pH}=2.0$, (d) $\mathrm{pH}=4.0\left(\mathrm{PO}_{4} /\right.$ Eu molar ratio of 200$)$. 
the as-synthesized products prepared with the different reactant $\mathrm{PO}_{4} / \mathrm{Eu}$ molar ratios and different $\mathrm{pH}$ values. The typical XRD pattern of the products prepared at the reactant $\mathrm{PO}_{4} / \mathrm{Eu}$ molar ratio of $60(\mathrm{pH}$ value of 0.70$)$ is shown in Fig. 1a. All the diffraction peaks agree well with hexagonal phase $\mathrm{EuPO}_{4}$ (JCPDS 20-1044). The diffraction peaks are very strong and sharp, indicating that the samples have a good crystallinity. While the reactant $\mathrm{PO}_{4} / \mathrm{Eu}$ molar ratio is 200 and the $\mathrm{pH}$ value is 0.85 , the diffraction peaks still agree well with hexagonal phase $\mathrm{EuPO}_{4}$ (Fig. 1b). When the reactant $\mathrm{PO}_{4} / \mathrm{Eu}$ molar ratio is 200, using ammonia (25\%) to adjust the $\mathrm{pH}$ values from 2.0 to 4.0 , all reflection peaks in Fig. 1c and d can be indexed to hexagonal phase $\mathrm{EuPO}_{4}$.

The morphology of the as-synthesized products prepared with different reactant $\mathrm{PO}_{4} / \mathrm{Eu}$ molar ratios and different $\mathrm{pH}$ values were investigated using SEM. As can be seen from Fig. 2a, the product synthesized with the reactant $\mathrm{PO}_{4} / \mathrm{Eu}$ molar ratio of 60 is uniform cluster-like nanostructures composed of nanowires, which has a diameter of about $80-90 \mathrm{~nm}$ and a length of about $1 \mu \mathrm{m}$. When the $\mathrm{PO}_{4} /$ Eu molar ratio increased to 200, the as-synthesized $\mathrm{EuPO}_{4}$ exhibits uniform flower-like microcluster morphology (Fig. 2b). The flower-like microstructures are actually composed of a self-assembly of the similar hexagonal prisms with a diameter of about $250 \mathrm{~nm}$ and a length of about $1.5 \mu \mathrm{m}$, which radiated out from the centers and formed uniform flower-like aggregates. Obviously, the concentration of phosphoric acid might be responsible for the morphologies formation of the as-synthesized nano/microstructured $\mathrm{EuPO}_{4}$. When phosphoric acid is excessive, the $\mathrm{pH}$ value of the reaction solution will decrease. To investigate the influence of $\mathrm{pH}$ values on the morphology, the products subjected to different $\mathrm{pH}$ values were studied by SEM (Fig. 2c and d). When the reactant $\mathrm{PO}_{4} / \mathrm{Eu}$ molar ratio is 200 , the $\mathrm{pH}$ value was adjusted to 2.0 by adding ammonia (25\%), the flower-like nanostructures composed of a large number of nanowires with a diameter of about $90-100 \mathrm{~nm}$ and a length of about $1-2 \mu \mathrm{m}$ were observed in
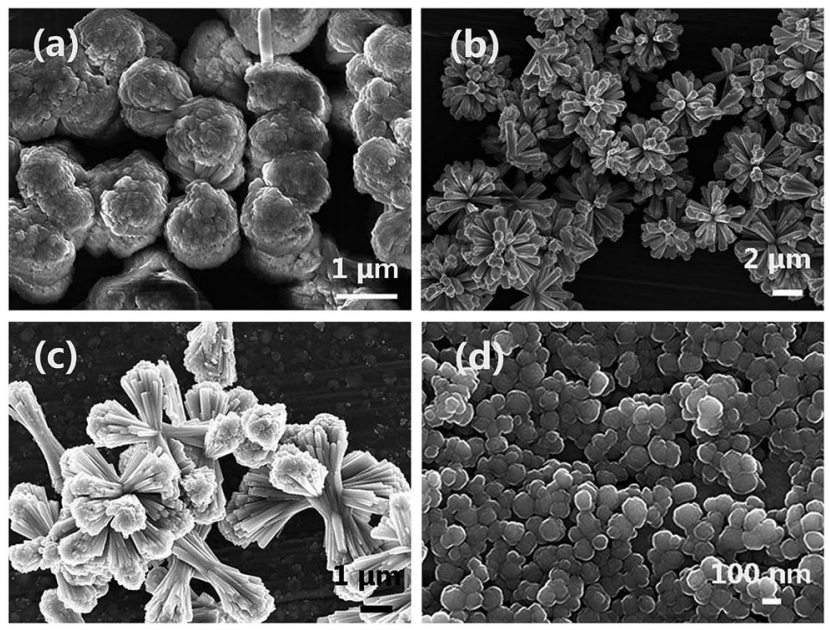

Fig. $2 \mathrm{SEM}$ images of $\mathrm{EUPO}_{4}$ nano/microstructures prepared with the different reactant $\mathrm{PO}_{4} /$ Eu molar ratios of (a) 60, (b) 200, and different $\mathrm{pH}$ values (c) $\mathrm{pH}=2.0$, (d) $\mathrm{pH}=4.0\left(\mathrm{PO}_{4} /\right.$ Eu molar ratio of 200$)$. the product (Fig. 2c). Subsequently, the $\mathrm{pH}$ values increased to 4.0 , the morphology of the product changed into uniform sphere-like nanostructures, which have a diameter of about 80$100 \mathrm{~nm}$ (Fig. 2d). In our system, when the $\mathrm{pH}$ value of the reaction solution is lower 2.0, the flower-like nanostructures composed of a large number of nanowires were obtained. When the $\mathrm{pH}$ values increased to 4.0, uniform sphere-like nanostructures were obtained. Fang et $a .^{32}$ reported that the hexagonal crystal structures are characteristic of highly anisotropic growth, a higher growth rate along the $c$ axis and a lower one perpendicular to the $c$ axis to form $\mathrm{LnPO}_{4}$ nanowires/nanorods. Our experimental results indicate that the $\mathrm{pH}$ value of the reaction solution is lower 2.0, the growth rate of $\mathrm{EuPO}_{4}$ along $c$ axis is faster than that in other directions, which results in the formation of $\mathrm{LnPO}_{4}$ nanorods/nanowires. When the $\mathrm{pH}$ values increased to 4.0, the $c$ axis direction of growth and the anisotropy is inhibited, and the formed nanorods/nanowires started to break up, and started to form nanoparticles. ${ }^{33}$

\subsection{Structure and morphology of core-shell $\mathrm{SiO}_{2} @ \mathrm{EuPO}_{4}$ nanostructures}

The SEM images of the as-synthesized $\mathrm{SiO}_{2}$ submicro-spheres and core-shell $\mathrm{SiO}_{2} @ \mathrm{EuPO}_{4}$ nanostructures are shown in the Fig. 3. The uniform-size $\mathrm{SiO}_{2}$ with an extremely smooth surface is first successfully prepared, which has a diameter of about $200 \mathrm{~nm}$ (Fig. 3a and b). Then the core-shell $\mathrm{SiO}_{2} @ \mathrm{EuPO}_{4}$ were prepared using TEP and $\mathrm{Eu}^{3+}$ ions as precursor. The $\mathrm{EuPO}_{4}$ nanoparticles were absorbed on the surface of the $\mathrm{SiO}_{2}$ submicro-spheres by the interactions between the $\mathrm{Si}-\mathrm{OH}$ and $\mathrm{EuPO}_{4}$. The surface of the core-shell $\mathrm{SiO}_{2} @ \mathrm{EuPO}_{4}$ is rough and has obvious granular substances, which might be caused by the inhomogeneous crystals of $\mathrm{EuPO}_{4}$ coating on the surface of $\mathrm{SiO}_{2}$ submicro-spheres. The core-shell $\mathrm{SiO}_{2} @ \mathrm{EuPO}_{4}$ nanostructures was further characterized by TEM analysis. The typical TEM images are shown in Fig. 4, which reveals that $\mathrm{SiO}_{2} @ \mathrm{EuPO}_{4}$ has a "core-shell" structure and present a uniform spherical morphology. Besides, $\mathrm{SiO}_{2} @ \mathrm{EuPO}_{4}$ has a $\mathrm{SiO}_{2}$ sphere with
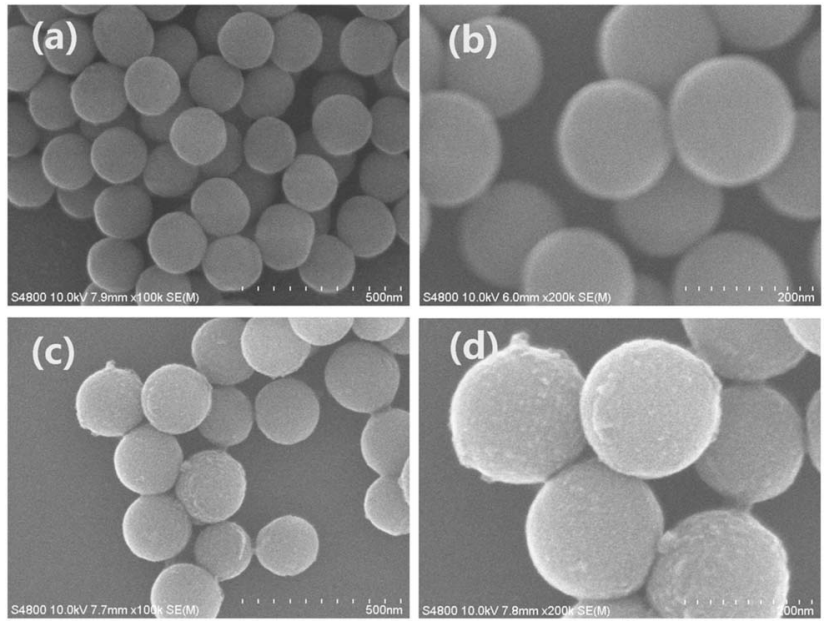

Fig. $3 \mathrm{SEM}$ images of $\mathrm{SiO}_{2}$ submicro-spheres (a, b), core-shell $\mathrm{SiO}_{2} \mathrm{aEuPO}_{4}$ nanostructures (c, d). 

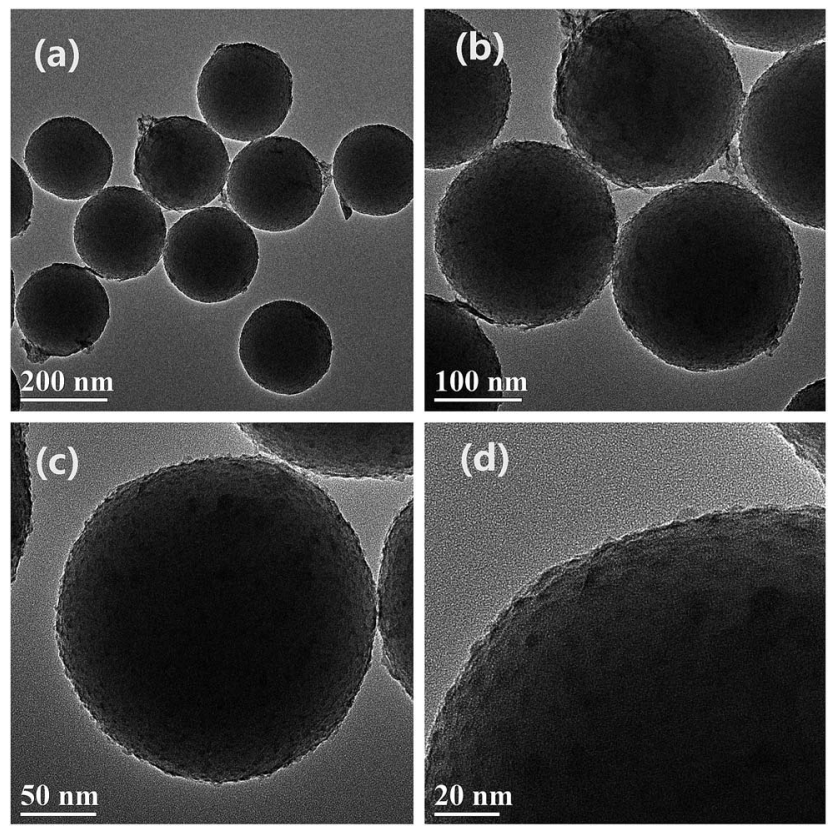

Fig. 4 TEM images of core-shell $\mathrm{SiO}_{2} \mathrm{aEuPO}_{4}$ nanostructures.

diameter about $200 \mathrm{~nm}$ and a thin $\mathrm{EuPO}_{4}$ surface. Further observation (Fig. 4d) indicated that the $\mathrm{EuPO}_{4}$ surface layer is composed by a number of small nanoparticles with a diameter of about $4 \mathrm{~nm}$, which accounts for the rough surface of $\mathrm{SiO}_{2} @ \mathrm{EuPO}_{4}$ spheres. The EDS spectrum of core-shell $\mathrm{SiO}_{2} @$ $\mathrm{EuPO}_{4}$ nanostructures is plotted in Fig. 5, which confirm the presence of $\mathrm{Eu}, \mathrm{O}, \mathrm{P}$ and $\mathrm{Si}$ in the thin layer of submicrospheres. The EDX mapping images are presented in Fig. S1. $\dagger$ As evidenced by the EDX mapping images, the elements of Eu, $\mathrm{P}, \mathrm{O}$, and $\mathrm{Si}$ are distributed uniformly in the single $\mathrm{SiO}_{2} @ \mathrm{EuPO}_{4}$ submicro-spheres. Further elemental analysis of the $\mathrm{SiO}_{2} @$ $\mathrm{EuPO}_{4}$ surface was performed by X-ray photoelectron spectroscopy (XPS). Fig. S $2 \uparrow$ shows the XPS spectrum of the core-shell $\mathrm{SiO}_{2} @ \mathrm{EuPO}_{4}$ nanostructures. The binding energies are calibrated using the carbon peak (C 1s) at $284.8 \mathrm{eV}$ as reference. As shown in the XPS spectrum, Eu, P, O, Si and C elements are detected. The peaks that located at 1135.0, 532.5, 284.8, 136.2,

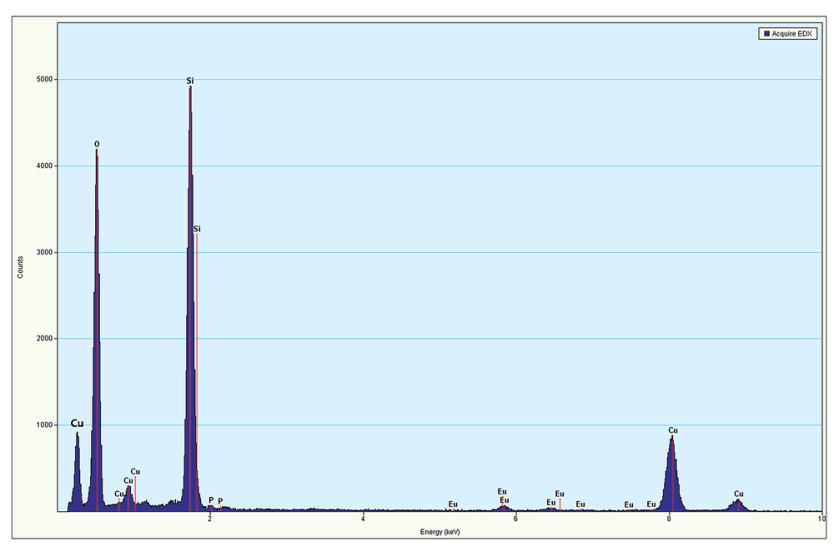

Fig. 5 EDS spectrum of core-shell $\mathrm{SiO}_{2}\left(\mathrm{aEuPO}_{4}\right.$ nanostructures. and $103.0 \mathrm{eV}$ can be attributed to the binding energy of Eu 3d, O 1s, C 1s, P 2p, Si 2p of the $\mathrm{SiO}_{2} @ \mathrm{EuPO}_{4}$ core-shell nanostructures, respectively. ${ }^{\mathbf{3 0 , 3 4}}$ The structures of the core-shell $\mathrm{SiO}_{2} @ \mathrm{EuPO}_{4}$ were further analyzed by the IR spectra (Fig. S3 $\dagger$ ). Fig. S3a $\dagger$ is IR spectrum of the $\mathrm{SiO}_{2}$ submicro-spheres. The broad band located at $3423 \mathrm{~cm}^{-1}$ is assigned to the $\mathrm{O}-\mathrm{H}$ stretching vibrations, and the peak at $1630 \mathrm{~cm}^{-1}$ is assigned to the bending vibrations of $\mathrm{O}-\mathrm{H}$ bond, which indicate that high content $\mathrm{OH}$ groups exist on the surface of the $\mathrm{SiO}_{2}$ submicrospheres. The surface $\mathrm{Si}-\mathrm{OH}$ groups play an important role in the formation of $\mathrm{EuPO}_{4}$ layer on the $\mathrm{SiO}_{2}$ submicro-spheres surfaces. The strong and wide absorption band located at $1104 \mathrm{~cm}^{-1}$ is attributed to the asymmetrical stretching vibration of $\mathrm{Si}-\mathrm{O}-\mathrm{Si}$, and the peak located at $798 \mathrm{~cm}^{-1}$ is assigned to symmetrical vibration of $\mathrm{Si}-\mathrm{O}-\mathrm{Si}$ bond. The peaks at $946 \mathrm{~cm}^{-1}$ and $465 \mathrm{~cm}^{-1}$ are assigned to symmetrical stretching vibration, and bending vibrations of $\mathrm{Si}-\mathrm{O}$ bond, respectively. IR spectrum of the core-shell $\mathrm{SiO}_{2} @ \mathrm{EuPO}_{4}$ nanostructures is showed in Fig. S3b. $\uparrow$ The peaks of $\mathrm{OH}$ groups on the surface of the $\mathrm{SiO}_{2}$ submicro-spheres also located at $3429 \mathrm{~cm}^{-1}$ and $1631 \mathrm{~cm}^{-1}$. However, the peak intensity is greatly weakened, which should result from the $\mathrm{EuPO}_{4}$ surface coating on the $\mathrm{SiO}_{2}$ submicro-spheres. Furthermore, a sharp peak at $1103 \mathrm{~cm}^{-1}$ appeared, which is the typical $\mathrm{P}-\mathrm{O}$ stretching vibrations originating from the thin shell layer of $\mathrm{EuPO}_{4}$ nanostructures. The other new peak at $470 \mathrm{~cm}^{-1}$ is assigned to $\mathrm{Eu}-\mathrm{O}$ bond..$^{35}$ The above results indicate that the $\mathrm{EuPO}_{4}$ are successfully coated on the $\mathrm{SiO}_{2}$ submicro-spheres.

Therefore, we suggest a possible formation mechanism of the core-shell $\mathrm{SiO}_{2} @ \mathrm{EuPO}_{4}$ nanostructures (Fig. 6) based on the above results. First, the $\mathrm{PO}_{4}{ }^{3-}$ ions were formed in the solution via the hydrolysis of TEP. EuPO ${ }_{4}$ were then formed by reaction of $\mathrm{PO}_{4}{ }^{3-}$ ions with europium ions. Finally, the $\mathrm{EuPO}_{4}$ were slowly absorbed on the surface of $\mathrm{SiO}_{2}$ submicro-spheres through a hydrogen bond or electrostatic adsorption interactions. It is assumed that TEP has two effects in the core-shell nanostructure formation process. On the one hand, it was a $\mathrm{PO}_{4}{ }^{3-}$ precursor by slowly hydrolyzing to provide $\mathrm{PO}_{4}{ }^{3-}$ ions. On the other hand, the slow release of $\mathrm{PO}_{4}{ }^{3-}$ ions can decrease the reaction rate of $\mathrm{PO}_{4}{ }^{3-}$ ions reacting with $\mathrm{Eu}^{3+}$ ions. It is quite possible that the $\mathrm{EuPO}_{4}$ nanoparticles are absorbed on the surface of the $\mathrm{SiO}_{2}$ submicrospheres at the early stage of the reactions, due to the weak interactions between the $\mathrm{Si}-\mathrm{OH}$ and $\mathrm{PO}_{4}{ }^{3-}$. With the extension of the reaction time, the $\mathrm{EuPO}_{4}$ nanoparticles gradually deposit on and coat on the surface of $\mathrm{SiO}_{2}$ submicro-spheres.

\subsection{Photoluminescence properties}

The photoluminescence (PL) spectra were measured at room temperature. Fig. $\mathrm{S} 4 \uparrow$ and 7 are the excitation and emission

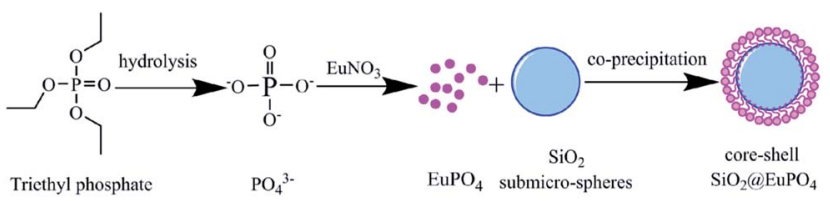

Fig. 6 The formation mechanism of the core-shell $\mathrm{SiO}_{2}\left(\mathrm{aEuPO}_{4}\right.$ nanostructures. 


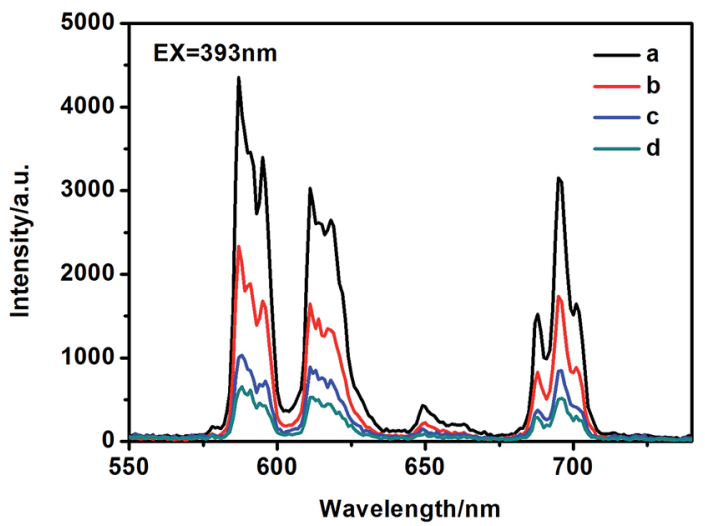

Fig. 7 Emission spectra of $\mathrm{EuPO}_{4}$ nano/microstructures prepared with a different reactant $\mathrm{PO}_{4} / \mathrm{Eu}$ molar ratios of (a) 60 , (b) 200, and different $\mathrm{pH}$ values (c) $\mathrm{pH}=2.0$, (d) $\mathrm{pH}=4.0\left(\mathrm{PO}_{4} /\right.$ Eu molar ratio of 200).

spectra of the cluster-like, flower-like and spherical-like $\mathrm{EuPO}_{4}$ nano/microstructures, respectively. The excitation peak centered at $393 \mathrm{~nm}$ could be contributed to the $\mathrm{f} \rightarrow \mathrm{f}$ transitions of the europium ions. ${ }^{36}$ The emission spectra shown in Fig. 7 is made up of four sharp peaks at 587, 610, 651, and $699 \mathrm{~nm}$, which correspond to the ${ }^{5} \mathrm{D}_{0} \rightarrow{ }^{7} \mathrm{~F}_{1},{ }^{5} \mathrm{D}_{0} \rightarrow{ }^{7} \mathrm{~F}_{2},{ }^{5} \mathrm{D}_{0} \rightarrow{ }^{7} \mathrm{~F}_{3}$, and ${ }^{5} \mathrm{D}_{0} \rightarrow{ }^{7} \mathrm{~F}_{4}$ optical transitions of $\mathrm{Eu}^{3+}$, respectively. And the photoluminescence intensity of the magnetic-dipole transition of ${ }^{5} \mathrm{D}_{0} \rightarrow{ }^{7} \mathrm{~F}_{1}$ was stronger than that of the electronic-dipole transition of ${ }^{5} \mathrm{D}_{0} \rightarrow{ }^{7} \mathrm{~F}_{2}$. When the trivalent europium ion lies on an inversion center, it is well known that the electric-dipole transitions would be strictly forbidden due to the parity selection rules. In consequence, the magnetic-dipole transition of ${ }^{5} \mathrm{D}_{0} \rightarrow{ }^{7} \mathrm{~F}_{1}$ dominated $(587 \mathrm{~nm}) \cdot{ }^{37,38}$ By contrast, due to the perturbation of the crystal field and the change of the local site symmetry, the degeneracy of ${ }^{7} \mathrm{~F}_{J}(J=1-4)$ energy level was resolved, and then the emission peaks split to multi-peaks. The transition of ${ }^{5} \mathrm{D}_{0} \rightarrow{ }^{7} \mathrm{~F}_{1}$ can split at most to three emission peaks, and ${ }^{5} \mathrm{D}_{0} \rightarrow{ }^{7} \mathrm{~F}_{2}$ can split to five peaks at most. ${ }^{39}$

Fig. $55 \dagger$ and 8 presented the excitation and emission spectra of the core-shell $\mathrm{SiO}_{2} @ \mathrm{EuPO}_{4}$ nanostructures. When the coreshell $\mathrm{SiO}_{2} @ \mathrm{EuPO}_{4}$ nanostructures were excited in the UV $(393 \mathrm{~nm})$, the spectrum displays four characteristic emission lines ascribed to ${ }^{5} \mathrm{D}_{0} \rightarrow{ }^{7} \mathrm{~F}_{J}(J=1-4)$ transitions of $\mathrm{Eu}^{3+}$. Interestingly, the photoluminescence intensity of electronic-dipole transition of ${ }^{5} \mathrm{D}_{0} \rightarrow{ }^{7} \mathrm{~F}_{2}$ was stronger than the magneticdipole transition of ${ }^{5} \mathrm{D}_{0} \rightarrow{ }^{7} \mathrm{~F}_{1}$. It is well-known that the ${ }^{5} \mathrm{D}_{0} \rightarrow{ }^{7} \mathrm{~F}_{2}$ transition is more sensitive to the symmetry than the ${ }^{5} \mathrm{D}_{0} \rightarrow{ }^{7} \mathrm{~F}_{1}$ transition. The symmetry around the $\mathrm{Eu}^{3+}$ ions of the core-shell $\mathrm{SiO}_{2} @ \mathrm{EuPO}_{4}$ nanostructures decreases leading to the enhancement of the ${ }^{5} \mathrm{D}_{0} \rightarrow{ }^{7} \mathrm{~F}_{2}$ transition. ${ }^{37}$ The size of $\mathrm{EuPO}_{4}$ on the surface of as-synthesized $\mathrm{SiO}_{2}$ submicro-spheres is ultra small, which might result in the $\mathrm{Eu}^{3+}$ ions cannot occupy a lattice site with inversion symmetry. Yan et al. $^{40}$ reported that when the size of the $\mathrm{REPO}_{4}: \mathrm{Eu}$ nanocrystals decreased, the ratio of surface $\mathrm{Eu}^{3+}$ ions increased and therefore, the symmetry around the $\mathrm{Eu}^{3+}$ ions decreases, leading to the increase in $I_{610} / I_{590}$. It's more remarkable that the emission

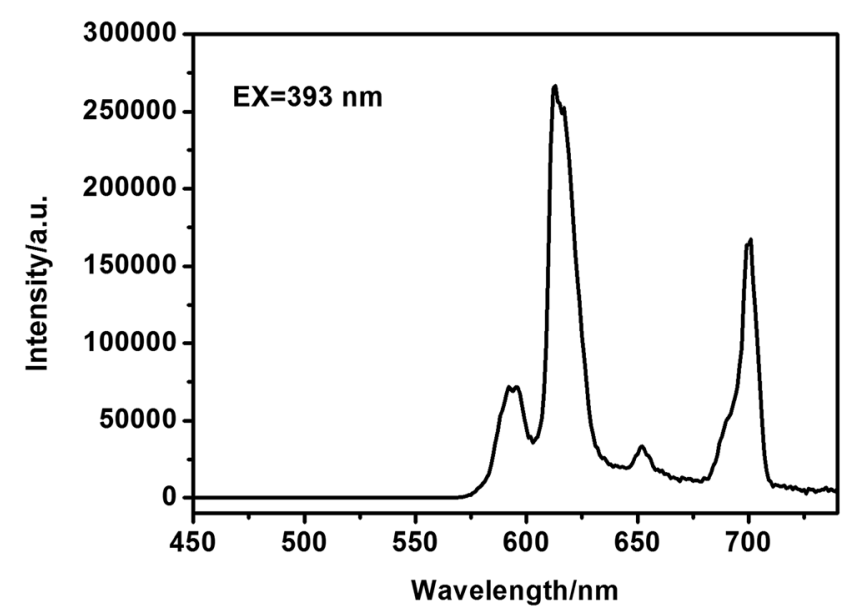

Fig. 8 Emission spectrum of the core-shell $\mathrm{SiO}_{2} \mathrm{aEuPO}_{4}$ nanostructures.

intensity of the core-shell $\mathrm{SiO}_{2} @ \mathrm{EuPO}_{4}$ nanostructures is 245600 a.u., which is 56.79 times higher than that of the flower-like EuPO $_{4}$ nanostructures. Comparing to the cluster-like, flower-like and spherical-like $\mathrm{EuPO}_{4}$ nano/microstructures, the core-shell $\mathrm{SiO}_{2} @ \mathrm{EuPO}_{4}$ nanostructures have excellent photoluminescence properties. The photoluminescence properties are affected by many factors including morphologies, sizes and crystal structures. The emission intensity of cluster-like morphologies composed by nanowires is stronger than that of spherical-like morphologies. In our previous work, it was found that the emission intensity of 1D nanorods/nanowires is stronger than that of nanoparticles. ${ }^{41} \mathrm{In}$ this study, when the $\mathrm{EuPO}_{4}$ nanoparticles coated on the surface of $\mathrm{SiO}_{2}$ submicro-spheres, the emission intensity of $\mathrm{Eu}^{3+}$ is stronger than that of $\mathrm{EuPO}_{4}$ particles with different morphology nano/ microstructures. We contribute this enhancement to the unique core-shell structure, in which the $\mathrm{SiO}_{2}$ cores greatly enhance the physical stability of $\mathrm{EuPO}_{4}$ and decrease the energy loss of $\mathrm{EuPO}_{4}$ molecular vibration.

The photoluminescence fitting curve of the core-shell $\mathrm{SiO}_{2} @ \mathrm{EuPO}_{4}$ nanostructures was also recorded, as shown in Fig. S6. $\uparrow$ It is noted that the decay curve can be fitted well with a biexponential function.

$$
I(t)=I_{0}+A_{1} \exp \left(-t_{1} / \tau_{1}\right)+A_{2} \exp \left(-t_{2} / \tau_{2}\right)
$$

Two lifetimes of $238.828 \mu$ s and $432.030 \mu$ s are detected. Therefore, the average lifetime $(\tau)$ is calculated using the following equation.

$$
(\tau)=\left(A_{1} \tau_{1}^{2}+A_{2} \tau_{2}^{2}\right) /\left(A_{1} \tau_{1}+A_{2} \tau_{2}\right)
$$

Herein, the calculated average lifetime $(\tau)$ is $374.494 \mu$ s of $\mathrm{Eu}^{3+}$ ion for the core-shell nanostructures. And these core-shell $\mathrm{SiO}_{2} @ \mathrm{EuPO}_{4}$ nanostructures with improved photoluminescence have possible applications for making optical devices namely upconvertors, LEDs, optical bioprobes, and so on. 


\section{Conclusions}

The cluster-like, flower-like and spherical-like $\mathrm{EuPO}_{4}$ nano/ microstructures, and the uniform core-shell $\mathrm{SiO}_{2} @ \mathrm{EuPO}_{4}$ nanostructures have been successfully synthesized by a hydrothermal route and a simple co-precipitation method, respectively. The cluster-like, flower-like and spherical-like $\mathrm{EuPO}_{4}$ nano/microstructures can be synthesized in a controlled manner by adjusting the reactant $\mathrm{PO}_{4} / \mathrm{Eu}$ molar ratios and the solution $\mathrm{pH}$ values. The core-shell $\mathrm{SiO}_{2} @ \mathrm{EuPO}_{4}$ nanostructures were achieved by using TEP and $\mathrm{Eu}^{3+}$ ions as precursors by the interactions between the $\mathrm{Si}-\mathrm{OH}$ and $\mathrm{EuPO}_{4}$. In addition, the photoluminescence properties demonstrate that the core-shell $\mathrm{SiO}_{2} @ \mathrm{EuPO}_{4}$ nanostructures have stronger emission intensity than the cluster-like, flower-like and spherical-like $\mathrm{EuPO}_{4}$ nano/ microstructures. Moreover, the electronic-dipole transition ${ }^{5} \mathrm{D}_{0} \rightarrow{ }^{7} \mathrm{~F}_{2}$ was stronger than the magnetic-dipole transition ${ }^{5} \mathrm{D}_{0} \rightarrow{ }^{7} \mathrm{~F}_{1}$, which indicates that the symmetry around the $\mathrm{Eu}^{3+}$ ions occupy low symmetry sites after the formation of the coreshell structures. This research has opened new opportunities not only in lowing the waste of precious phosphors by coating the rare earth phosphate on the $\mathrm{SiO}_{2}$, and but also in developing advanced luminescence materials with improved photoluminescence properties.

\section{Conflicts of interest}

There are no conflicts to declare.

\section{Acknowledgements}

This work is supported by the Major projects of Natural Science Foundations of Inner Mongolia Science Foundation (2015ZD01) and the Natural Science Foundations of Inner Mongolia Science Foundation (2015MS0502).

\section{Notes and references}

1 M. Yu, J. Lin and J. Fang, Chem. Mater., 2005, 17, 1783-1791.

2 H. Ding, H. S. Tao, X. Z. Zhu and M. G. Li, Appl. Surf. Sci., 2017, 41, 9580-9585.

3 A. A. Markeb, A. Alonso, A. Sánchez and X. Font, Sci. Total Environ., 2017, 598, 949-958.

4 S. Y. Gim, S. Hong, J. Kim, Y. J. Kwon, M. Kim, G. H. Kim and J. H. Lee, Food Chem., 2017, 235, 160-166.

5 W. Di, X. Zhao, S. Lu, X. Wang and H. Zhao, J. Solid State Chem., 2007, 180, 2478-2484.

6 Y. H. Haba, C. Kojima, A. Harada and K. Kono, Macromolecules, 2006, 39, 7451-7453.

7 F. Caruso, A. S. Susha, M. Giersig and H. Mohwald, Adv. Mater., 1999, 11, 950-953.

8 S. L. Westcott, S. J. Oldenburg, L. Randall and N. J. Halas, Langmuir, 1998, 14, 5396-5401.

9 P. D. Yang, D. Y. Zhao, D. I. Margolese, B. F. Chmelka and G. D. Stucky, Nature, 1998, 396, 152-155.

10 K. Suzuki, Y. Saito, Y. Tokuoka, M. Abe and T. Sato, J. Am. Chem. Soc., 1997, 74, 55-59.
11 D. L. Berthier, I. Schmidt, W. Fieber, C. Schatz, A. Furrer, K. Wang and S. Lecommandoux, Langmuir, 2010, 26, 79537961.

12 D. L. Berthier, A. Herrmann and L. Ouali, Polym. Chem., 2011, 2, 2093-2101.

13 M. Ocaña, E. Cantelar and F. Cussó, Mater. Chem. Phys., 2011, 125, 224-230.

14 L. Liu, H. Xiao, X. An, Y. Zhang, R. Qin, L. Liu, D. Zhang, R. Sun and L. Chen, Chem. Phys. Lett., 2015, 619, 169173.

15 W. X. Li, Y. S. Zheng, X. F. Cao, J. Bai, Z. F. Fu and J. R. Bao, J. Lumin., 2016, 178, 470-478.

16 T. S. Atabaev, O. S. Jin, J. H. Lee, D. W. Han, H. H. T. Vu, Y. H. Hwang and H. K. Kim, RSC Adv., 2012, 2, 9495-9501.

17 H. Wang, M. Yu, C. K. Lin, X. M. Liu and J. Lin, J. Phys. Chem. $C, 2007,111,11223-11230$.

18 M. Yu, J. Lin and J. Fang, Chem. Mater., 2005, 17, 17831791.

19 F. Zhang and S. Wong Stanislaus, ACS Nano, 2010, 4, 99-112. 20 S. Rodriguez-Liviano, A. I. Becerro, D. Alcántara, V. Grazú, J. M. de la Fuente and M. Ocaña, Inorg. Chem., 2013, 52, 647-654.

21 W. Di, X. Zhao, S. Lu, X. Wang and H. Zhao, J. Solid State Chem., 2007, 180, 2478-2484.

22 Y. C. Kang, E. J. Kim, D. Y. Lee and H. D. Park, J. Alloys Compd., 2002, 347, 266-270.

23 B. Moine and G. Bizarri, Opt. Mater., 2006, 28, 58-63.

24 A. K. Parchur and R. S. Ningthoujam, RSC Adv., 2012, 2, 10854-10858.

25 O. Lehmann, K. Kompe and M. Haase, J. Am. Chem. Soc., 2004, 126, 14935-14942.

26 X. Li and H. F. Bi, J. Alloys Compd., 2012, 532, 72-77.

27 R. S. de-Oliveira, B. S. de-Brito, J. Kulesza, S. Alves-Jr and B. S. Barros, Ceram. Int., 2017, 43, 8276-8283.

28 G. G. Li, L. L. Li, M. M. Li, Y. H. Song, H. F. Zou, L. C. Zou, X. C. Xu and S. C. Gan, Mater. Chem. Phys., 2012, 133, 263268.

29 M. Yu, H. Wang, C. K. Lin, G. Z. Li and J. Lin, Nat. Nanotechnol., 2006, 17, 3245-3252.

30 J. Dai, M. Lv, G. Chao and X. Li, Mater. Des., 2015, 83, 795800.

31 W. Stöber, Inhalation Toxicol., 1999, 11, 269-292.

32 Y. P. Fang, A. W. Xu, R. Q. Song, H. X. Zhang, L. P. You, J. C. Yu and H. Q. Liu, J. Am. Chem. Soc., 2003, 125, 1602516034.

33 T. T. Huong, L. T. Vinh, T. K. Anh, H. T. Khuyen, H. T. Phuong and L. Q. Minh, New J. Chem., 2014, 38, 2114-2119.

34 J. H. Lang, Q. Zhang, Q. Han, Y. Fang, J. Y. Wang, X. Y. Li, Y. Q. Liu, D. D. Wang and J. H. Yang, Mater. Chem. Phys., 2017, 194, 29-36.

35 M. Yu, J. Lin, J. Fu, H. J. Zhang and Y. C. Han, J. Mater. Chem., 2003, 13, 1413-1419.

36 T. S. Atabaev, H. T. V. Hong, H. K. Kim and Y. H. Hwang, J. Alloys Compd., 2012, 525, 8-13.

37 Y. G. Yang, Mater. Sci. Eng., B, 2013, 178, 807-810. 
38 C. Zollfrank, H. Scheel, S. Brungs and P. Greil, Cryst. Growth Des., 2008, 8, 766-770.

39 Y. H. Zheng, H. P. You, G. Jia, K. Liu, Y. H. Song, M. Yang and H. J. Zhang, Cryst. Growth Des., 2009, 9, 5101-5107.
40 H. X. Mai, Y. W. Zhang, L. D. Sun and C. H. Yan, Chem. Mater., 2007, 19, 4514-4522.

41 J. R. Bao, R. B. Yu, J. Y. Zhang, D. Wang, J. X. Deng, J. Chen and X. R. Xing, Scr. Mater., 2010, 62, 133-136. 